\title{
Antibiotics and renal branching morphogenesis: comparison of toxicities
}

\author{
Ruud R.G. Bueters' ${ }^{1}$, Lisanne J.A. Kusters' ${ }^{1}$, Annelies Klaasen' ${ }^{1}$, Lambertus P. van den Heuvel' and Michiel F. Schreuder ${ }^{1}$
}

BACKGROUND: Many premature born neonates receive antibiotic drugs to treat infections, which are applied during active nephrogenesis. We studied the impact of clinical concentrations of gentamicin and alternatives, ceftazidime and meropenem, on ureteric branching.

METHODS: Mice metanephroi were dissected at embryonic day 13 and cultured in media with or without various concentrations of gentamicin, ceftazidime, or meropenem. Zero and $24 \mathrm{~h}$ kidney size were assessed by surface area measurements, and the ureteric tree was visualized by whole mount staining and confocal microscopy. Branching was evaluated by counting and gene expression levels of Wt1, Sox9, Bmp7, Fgf8, and Gdnf were investigated.

RESULTS: A concentration of 2,000 $\mu \mathrm{mol} / \mathrm{l}$ ceftazidime impaired ureteric development. In addition, a 4.5-fold and a 2.5-fold downregulation was noted in Fgf8 and Gdnf, respectively. No adverse effects were noted after gentamicin or meropenem treatment. No relationship was noted between surface area expansion and ureteric bud formation, but surface area at explantation related to bud count after $24 \mathrm{~h}$ of culture. CONCLUSION: Ceftazidime, but not gentamicin or meropenem reduced ureteric branching in mice and suggest a role for Fgf8 and Gdnf in its mechanism. Metanephros surface area measurements can be used to reduce intra- and inter-litter variation.

K idney development, leading to the formation of nephrons, starts around the 5 th wk of gestation and terminates before term birth, around the 34th-36th wk of gestation. Many factors have been described to disturb this developmental process, leading to long-term problems such as hypertension and chronic kidney disease (1).

One such disturbing factor may be the use of (nephrotoxic) drugs during kidney development, such as in pregnant women or neonates born before termination of nephrogenesis. Gentamicin, as well as other aminoglycosides, is widely used as part of the first line treatment of (suspected) bacterial infection in neonates to combat Gram-negative infections. Based on data from the Netherlands Perinatal Registry, $62 \%$ of neonates born before $32 \mathrm{wk}$, who can be considered the most vulnerable group, are treated with aminoglycosides (2). However, due to the fact that gentamicin is classified as a nephrotoxic drug, controversy remains on its safety as aminoglycosides have been shown to disturb kidney development and lead to a reduced nephron number in some experimental animals (3-6) and organ culture studies (7).

The aim of our research was to study the impact of antibiotic treatments on nephrogenesis in a model of early nephrogenesis. Gentamicin was studied as well as clinically relevant, alternative drug treatments to compare the toxic potentials of these drugs in a clinical dose range. As alternative treatments, we chose a third generation cephalosporin, ceftazidime, and the carbapenem meropenem. These two drugs both have properties to deal with Gram-negative bacteria and have different mechanisms of action compared to gentamicin. Although beta-lactams have their own potencies to be nephrotoxic, ceftazidime can be given at a fairly high dose before proximal tubule damage is noticeable (8). As for meropenem, this drug is regarded as one of the safer carbapenems (9) due to its stability to renal dehydropeptidase-I (10), which was confirmed in a large clinical trial (11). Although these alternatives to gentamicin appear quite safe, information on the potential toxic effects on renal branching morphogenesis is lacking. We hypothesized that gentamicin would hamper early kidney development, and ceftazidime and meropenem would prove to be safe.

\section{RESULTS}

\section{Metanephric Growth}

First, we studied the usefulness of surface area growth as a good marker for renal development. Based on previous studies $(7,12,13)$, a relationship between metanephric size expansion and ureteric tip count, a more direct measurement of renal development, was expected. However, as can be noted in Figure 1a, surface area expansion did not correlate with ureteric tip formation $\left(R^{2}=0.005, P=0.77\right)$ and was therefore not used as a marker in our studies.

In addition, we noted a variation in metanephric size within a litter (up to $11 \%$ ) and between our litters ( $\pm 20 \%$ ), based on differences in surface area measured directly after explantation. The impact of this variation on the ureteric bud tip count after $24 \mathrm{~h}$ was studied. As can be seen in Figure 1b, a significant 
a

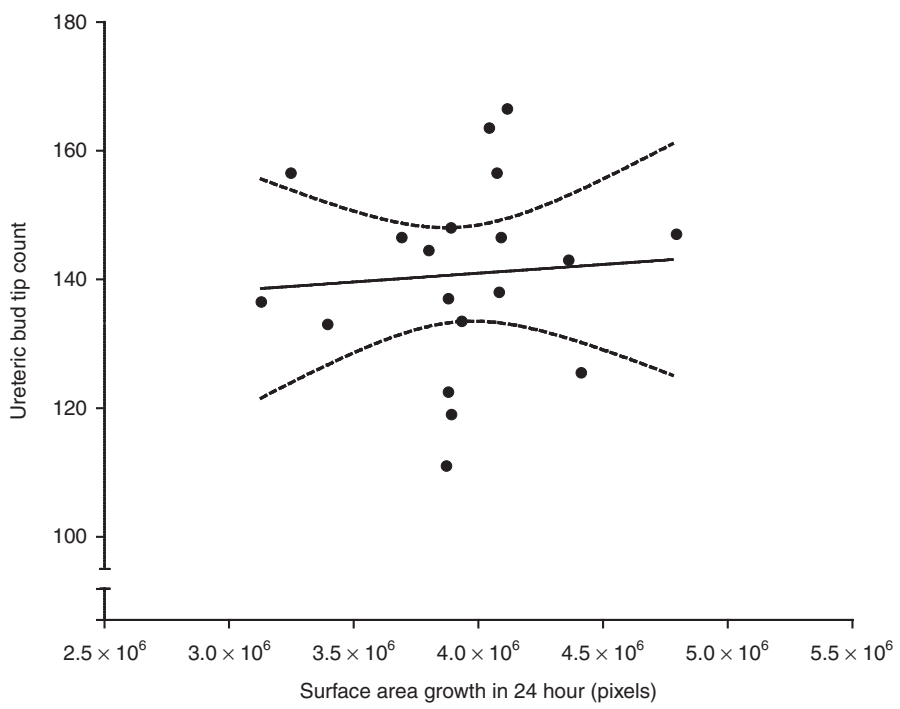

b

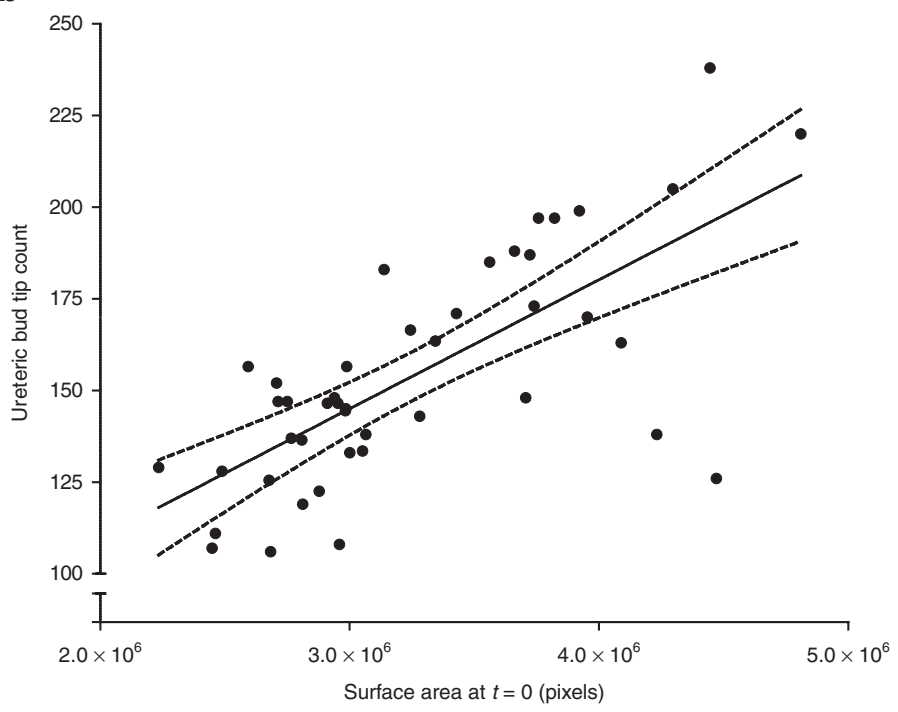

Figure 1. Correlation (with $95 \%$ confidence interval) between surface area measurements and ureteric bud formation (a) after $24 \mathrm{~h}$ of organ culture and (b) at explantation. Pearson's correlation coefficients were $R^{2}=0.005228$ and $R^{2}=0.5082$ for $\mathbf{a}$ and $\mathbf{b}$, respectively.

correlation was noted between metanephric size and ureteric bud development $\left(R^{2}=0.5, P<0.01\right)$. Based on this finding, all ureteric tip counts were corrected for metanephric surface area at explantation.

\section{Ureteric Tip Imaging}

Ureteric tip development was studied in the three different antibiotic classes. A range of three doses was tested to evaluate the broad drug response and investigate a possible dose response relationship (Figure 2).

As can be noted in Figure $\mathbf{2 b}$, only the 2,000 $\mu \mathrm{mol} / \mathrm{l}$ ceftazidime concentration resulted in a statistically significant lower ureteric tip count after $24 \mathrm{~h}$ of exposure (28.37 vs. $48.16, P<$ 0.01 ). This effect was also clearly detectable visually (Figure 3 ). No effects on development compared to control were noted at the lower and the middle dose. In our experiments with gentamicin (Figure 2a) a trend was noticeable towards a lower ureteric tip count. Meropenem treatment (Figure 2c) did not show an effect on ureteric tip development compared to control at any of the concentrations studied.

\section{Gene Expression Analysis}

We studied a selection of targets in known nephrogenesis and apoptosis pathways by mRNA expression analysis (Figure 4). Robust CT levels indicated that the targets were present in our metanephric organ cultures.

Treatment with ceftazidime in a concentration of 2,000 $\mu \mathrm{mol} / \mathrm{l}$ resulted in a 2.5 -fold downregulation of $G d n f$ mRNA, a well known factor in the ureteric branching pathway (14). In addition, a 4.5 -fold downregulation of the Fgf 8 mRNA was noted (Figure 4c). No changes in expression patterns were noted after treatment with $200 \mu \mathrm{mol} / \mathrm{l}$ of ceftazidime. Gentamicin or meropenem treatment did not have an effect on the mRNA levels of our chosen targets. Furthermore, mRNA levels of Caspase 3 and Caspase 9 remained similar between controls and high dose treatments of gentamicin and ceftazidime.

\section{DISCUSSION}

We have shown that an upper clinical range concentration of ceftazidime reduces ureteric branching morphogenesis and suggest a role for $F g f 8$ and $G d n f$ pathways in its toxicity. No adverse effects of gentamicin and meropenem within their postulated clinical dose range were noted on ureteric branching or basic nephrogenesis pathways.

Previous reports have used surface area expansion as a measurement for renal development $(7,12,13)$. Our study shows no correlation between the surface expansion and a more direct kidney developmental marker such as ureteric bud count. This lack of correlation may be explained by flattening of the metanephros in organ culture and its partial loss of three dimensional structure as has been reported previously (15). However, the impact on actual measurements had not been studied. We show that surface area measurements in the first $24 \mathrm{~h}$ after explantation are not a proper marker for renal development.

Although flattening of the organ rudiment during organ culture renders surface analysis unhelpful for quantification of kidney development, surface area at explantation can partially predict the amount of tips that will develop in metanephroi. We found a significant correlation between the surface area of explant size directly after dissection and the amount of ureter tips counted after $24 \mathrm{~h}$. One could expect such a correlation because kidney development is more pronounced in utero than in organ culture (16). Therefore, a more evolved (and therefore branched and larger) metanephros at time of dissection can be expected to have more end tips after $24 \mathrm{~h}$ of growth. These data allow for correction of variation in gestational age. By using our correlation to correct all ureteric tip counts for the measured surface area after explantation, variability of developmental stage will be more limited, and more precise 


\section{Articles $\mid$ Bueters et al.}

a

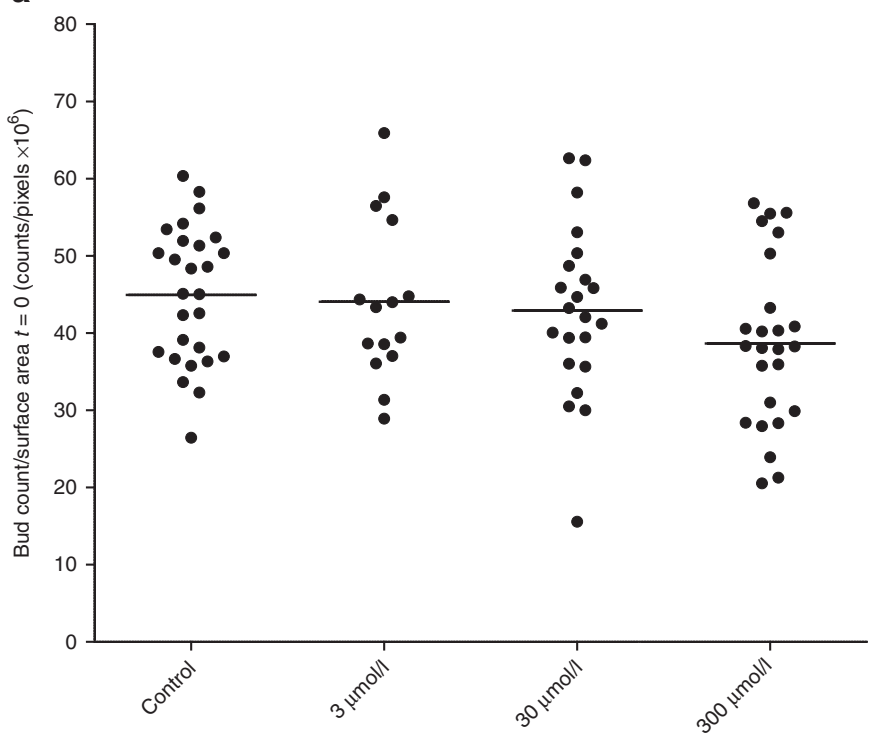

C b

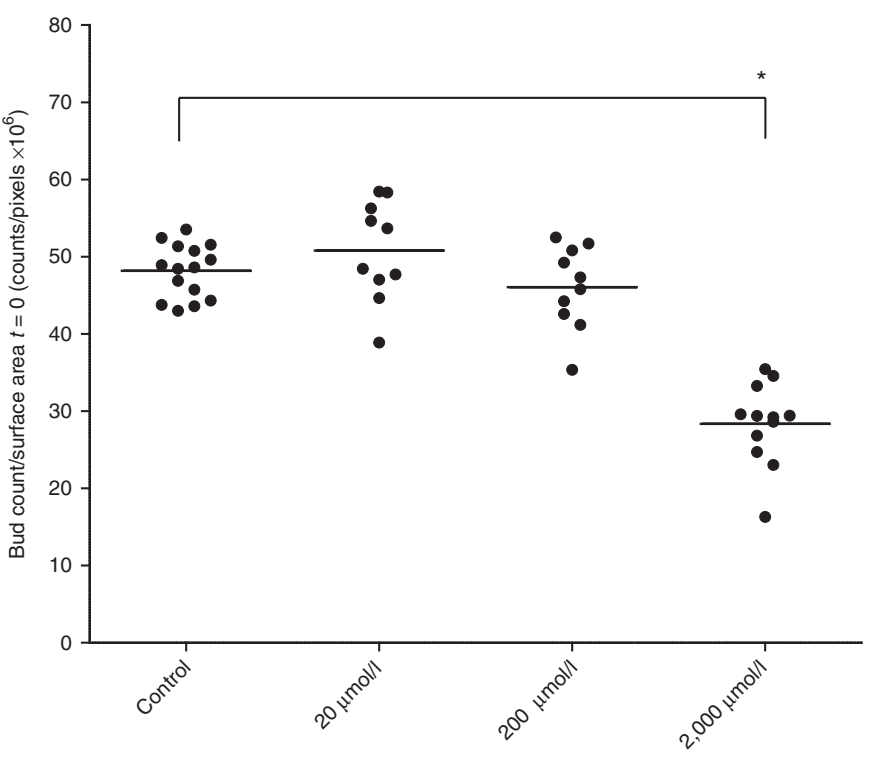

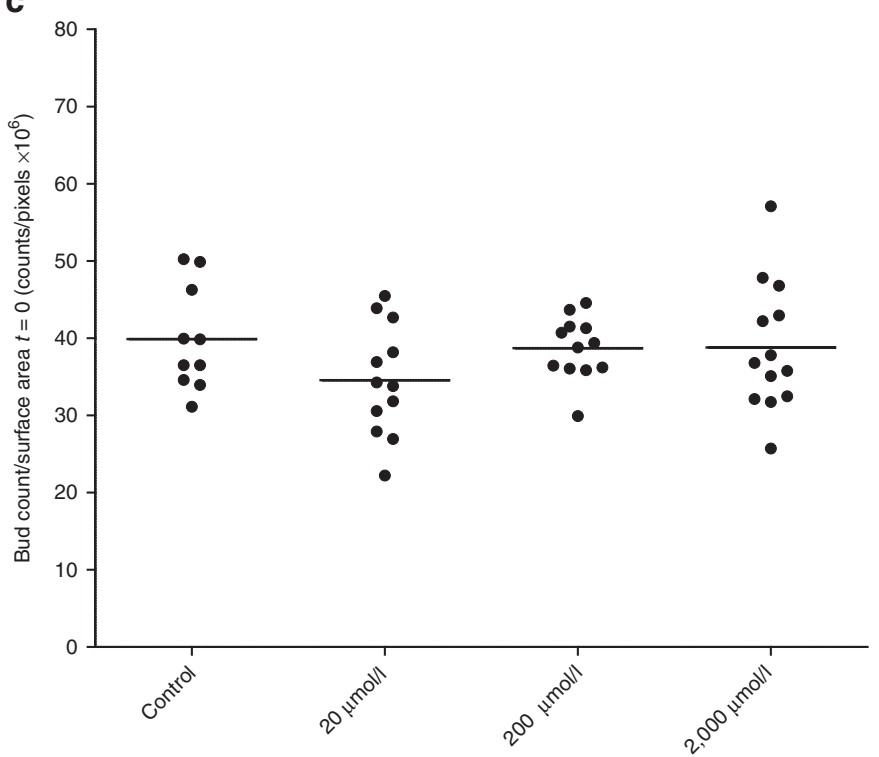

Figure 2. Quantitative analysis of ureteric buds in metanephroi cultured for $24 \mathrm{~h}$ in media with three antibiotic drugs, i.e., (a) gentamicin, (b) ceftazidime, and (c) meropenem. Individual data points as well as means per treatment group are presented. A low, clinical, and high concentration were tested. Sample sizes of treatment groups were between 15 and $27 ; 10$ and $15 ;$ and 10 and 13 for gentamicin, ceftazidime, and meropenem, respectively. ${ }^{*} P<0.01$.

comparisons between dose groups, but also between litters can be obtained.

Our initial research question was whether clinical relevant levels of gentamicin could influence nephrogenesis and whether other classes of antibiotics might be safer.

The potential of gentamicine to influence nephrogenesis has already been established in rat. Studies were mostly performed by either maternal dosing $(3-5)$ or postnatal dosing $(3,17)$. In these studies, no effect of gentamicin was noted on renal development after early postnatal dosing during active nephrogenesis. However, after maternal dosing gentamicin accumulation was confirmed in the fetal kidney. In addition, exposure to gentamicine in utero resulted in a reduction of glomerular numbers up to $20 \%$ and alterations in the tubular structure.
However, the dose administered to the pregnant females in these studies was $75 \mathrm{mg} / \mathrm{kg}$ and was almost 20 -fold higher than the $4 \mathrm{mg} / \mathrm{kg}$ clinical dose we extrapolated the dose levels from in this study.

Our results indicate that $24 \mathrm{~h}$ exposure of gentamicin did not result into impaired ureteric branching or altered expression of key pathways. Even though we did notice a trend towards reduced ureteric branching this difference was not statistically significant. This contradicts earlier findings of Gilbert et al. who found reduced ureteric branching in rat organ cultures at a gentamicin concentration of $100 \mu \mathrm{mol} / \mathrm{l}$ (7). The main difference between our approaches can be found in animal species (rat vs. mice in the current study) and in developmental stage. We believe the species difference is probably of secondary importance to the 


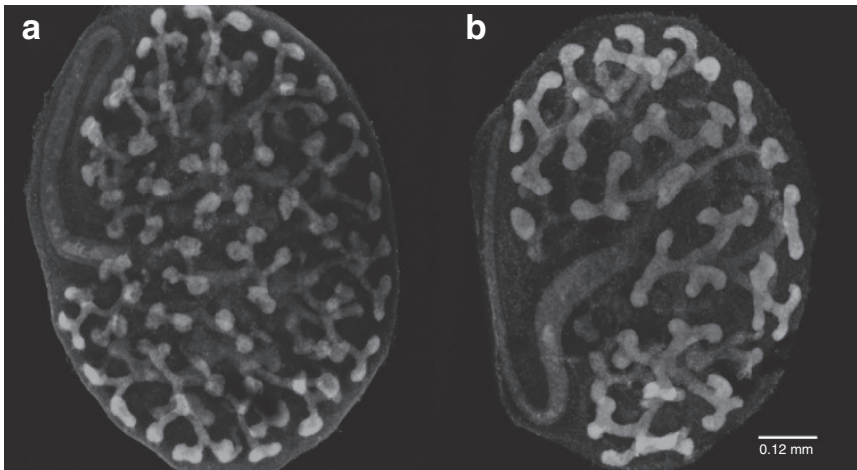

Figure 3. Representative immunohistochemic staining of ureteric bud development in metanephroi cultured for $24 \mathrm{~h}$ in media with (b) 2,000 $\mu \mathrm{mol} / \mathrm{l}$ ceftazidime or (a) vehicle control.

a

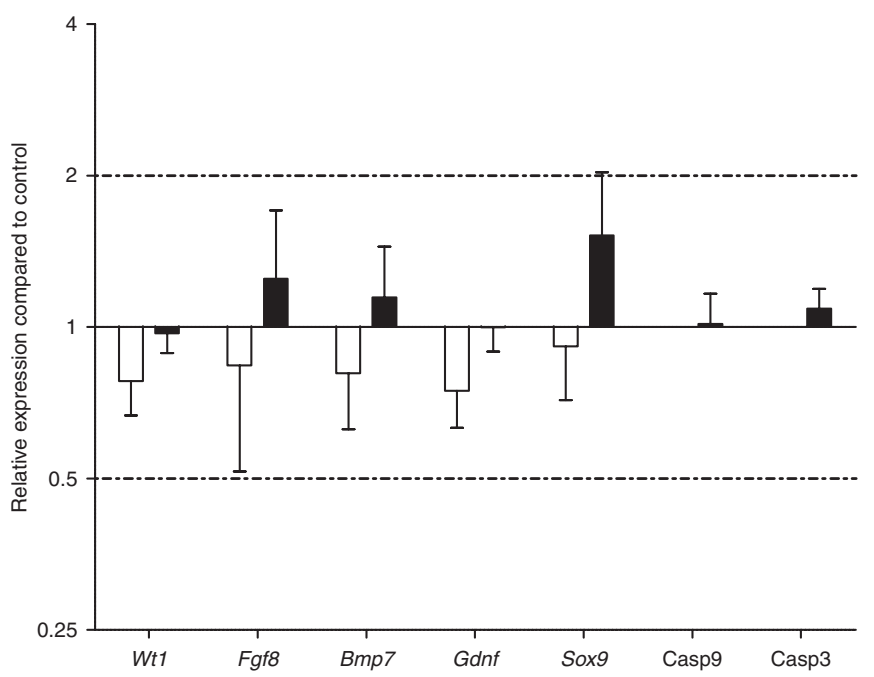

developmental stage as effects of in utero gentamicin exposure on renal development were reported in rats, mice and guinea pigs alike $(3-7,18)$. Although some differences in susceptibility may be present. Comparing Theiler and Witschi developmental stages (19), our E13 mice are approximately 12-24h more mature compared with the E14 rat embryos. It is well known that timing of insults during organ development is of great importance for the outcome and that dosing at a day later may completely abolish any effect (20). Taken these factors into account, gentamicin has the potential to disturb nephrogenesis, but the clinical dose range seemed to be safe when administered in the time frame and species that we studied.

From the data of our selected potential alternatives, we conclude that ceftazidime does not appear to be a good substitute for gentamicin. In the high dose group, an impaired kidney

b

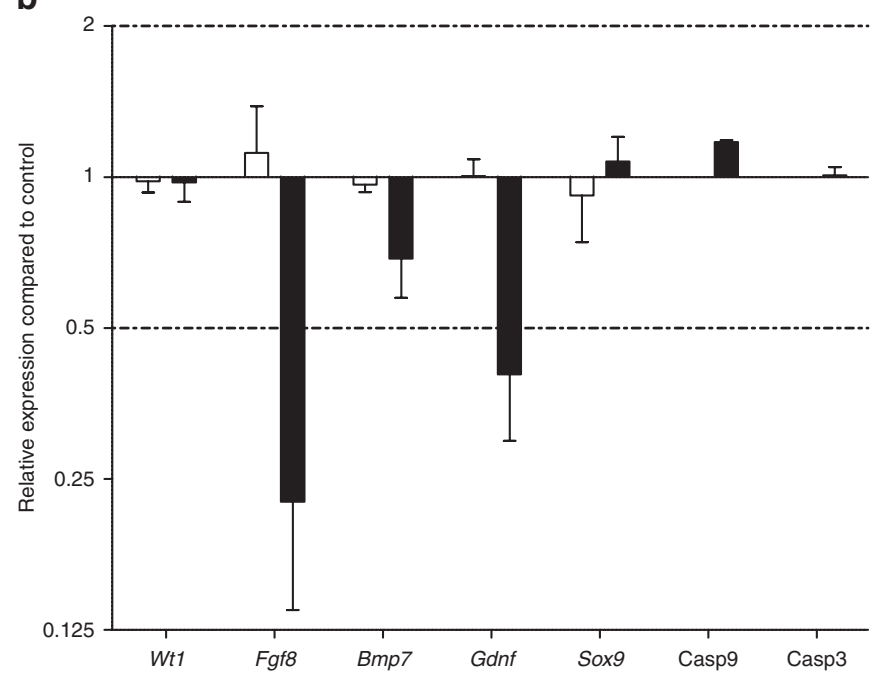

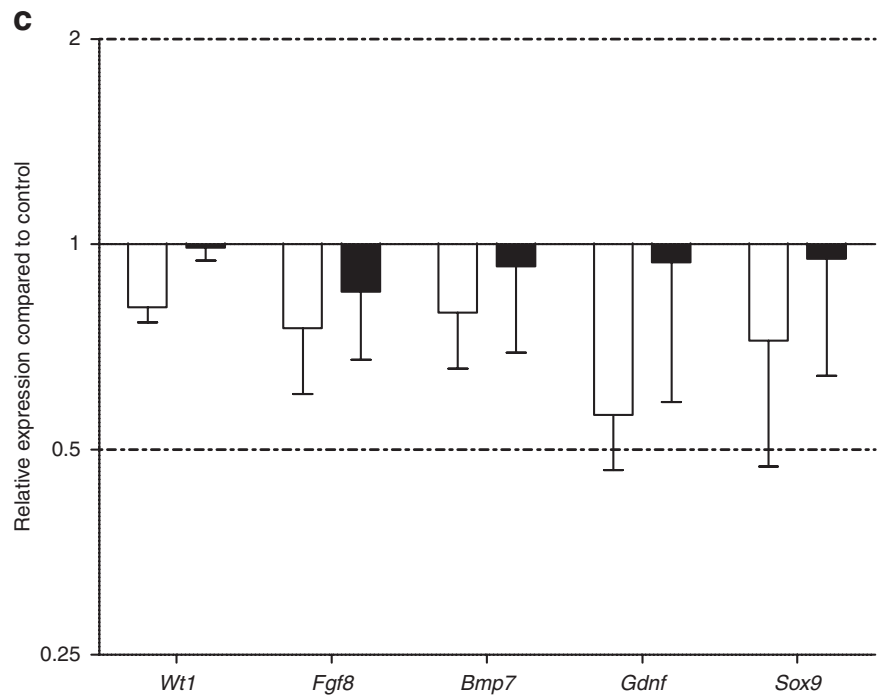

Figure 4. Gene expression of important nephrogenesis pathway targets in metanephroi cultured for $24 \mathrm{~h}$ in media with three antibiotic drugs, i.e., (a) gentamicin, (b) ceftazidime, and (c) meropenem. Means with SEM are presented for (a) two or (b,c) three experiments, with exception of the caspase targets, which were done in two experiments. Concentrations tested were 30 (white bars) and $300 \mu \mathrm{mol} / \mathrm{l}$ (black bars) for gentamicin, 200 (white bars) and 2,000 $\mathrm{mmol} / \mathrm{l}$ (black bars) for both ceftazidime and meropenem. Six to nine metanephroi were pooled per experiment. 
development and downregulation in corresponding pathways was identified.

We hypothesize that ceftazidime affects the renal progenitor cell population causing downregulation of $F g f 8$ and subsequent downregulation of $G d n f$ resulting in ureteric bud impairment. Fgf8 is important in cell survival of renal progenitor cells, which are an important source of Gdnf production $(21,22)$. In contrast, no expression changes were noted in $B m p 7$, which is also localized to the progenitor cell population (23), suggesting no general progenitor cell loss with possibly cell loss in a specific sub population. This finding is supported with the normal expression levels of Caspase 3 and Caspase 9, markers of apoptotic insults and also important in the normal development of the renal tubular network (24). Alternatively, ceftazidime may directly affect both the mesenchyme and the ureteric bud. Although this high dose is tenfold higher than our theoretical calculated clinical dose, the actual intracellular concentration in human cells is unknown. Based on our results, the safety of ceftazidime for treatment of pregnant women should be reconsidered, as it may be harmful for early renal development of the fetus. In addition, ceftriaxone was earlier reported to have a negative effect on nephrogenesis in rats (25), which underlines the general potential harmful effects of maternal cephalosporine use on fetal nephrogenesis.

In contrast, meropenem did not show any negative effect on ureteric bud branching or mRNA levels of important development pathways, and may therefore be a potential useful substitution for gentamicin with regards to nephrogenesis.

Our studies have some limitations. First, due to the stage of development and the method of antibody staining, we are currently unable to evaluate ureteric bud staining beyond $24 \mathrm{~h}$ of exposure. Therefore, it might be that prolonged exposure to gentamicin or meropenem will eventually result in renal damage. Using genetically modified mice with intrinsic fluorescent protein expression in the ureteric bud may solve this problem and allow for such longer-term studies (16). Second, we have no data on drug uptake within the cells. Gentamicin has been shown to be detectable within embryonic kidney cells after $8 \mathrm{~h}$ of exposure (7). Our ex vivo model is slightly more mature and of a different species which could have influence uptake. However, we noted a dose related trend in our experiments and are therefore confident that we had uptake of gentamicin in our model. For ceftazidime and meropenem data on uptake in organ culture are not yet available, which may play a role in the absence of effects in the meropenem treated metanephroi. However, the reduced formation of ureteric bud tips in the high dose ceftazidime group suggests uptake of ceftazidime by

Table 1. Dose selection parameters

\begin{tabular}{lccc}
\hline & Gentamicin & Ceftazidime & Meropenem \\
\hline (Neonatal) dose $(\mathrm{mg} / \mathrm{kg})$ & 4 & 50 & 20 \\
Molecular weight & 463.6 & 546.58 & 383.46 \\
Plasma binding $(\%)$ & 0 & 27 & 2 \\
Volume of distribution $(\mathrm{l} / \mathrm{kg})$ & 0.31 & 0.36 & 0.3 \\
\hline
\end{tabular}

Data in this table were derived from multiple sources (26-32). the metanephros within hours. Furthermore, we investigated a selection of targets in the nephrogenesis pathways and studied gene expression rather than protein expression. An immunohistochemical approach was attempted, but has not provided additional insight so far. Besides the technical difficulties of studying protein expression levels in small tissues as metanephroi, we feel that the significant morphological change in these cultured metanephroi show the relevance of the altered gene expression levels. In order to study apoptosis, we decided to study mRNA levels of 2 different caspases. Alternatively, TUNEL could be used to the same extent and should be performed in future studies. Finally, at present we do not have data on different time points during development at which the kidney may be more vulnerable to specific drugs. This would be a very important future step in relating study findings to the human embryonic kidney development.

Our objective was to determine whether these antibiotic compounds could interfere with a basal developmental process as ureteric bud differentiation when administered in a clinical dose range. Based on our data, we can conclude that short-term gentamicin or meropenem treatment at this stage of embryonic kidney development does not have an adverse impact on nephrogenesis. However, ceftazidime was shown to inhibit ureteric branching and the expression of Fgf8 and $G d n f$, two important players in kidney development. Furthermore, metanephric surface area growth should not be used as a renal developmental predictor, but surface area at explantation can be used to correct for intra- and inter-litter variation in developmental stages.

\section{METHODS}

\section{Drugs}

Gentamicin (G1272) and ceftazidime hydrate (C3809) were obtained from Sigma-Aldrich (Zwijndrecht, The Netherlands). Meropenem was obtained from Fresenius Kabi (Teramo, Italy).

\section{Organ Culture}

Experiments were approved by the Animal Ethics Committee at the Radboud University Nijmegen. Embryonic day 13 time pregnant HSD:ICR female mice (Harlan, Horst, The Netherlands) were euthanized after arrival by cervical dislocation and kidneys were dissected from the embryos by means of two small needles. Intact isolated metanephroi were held on ice in pre-cooled Leibovitz medium (Gibco, Paisley, UK) until transfer to the organ culture system. Metanephroi were cultured on $0.4 \mu \mathrm{m}$ pore size Millicell cell culture inserts (Millipore, Carrigtwohill, Ireland) placed in a six-well plate containing DMEM/F-12 (1:1) medium (Gibco), supplemented with $10 \mathrm{mg} / \mathrm{l}$ insulin, $5,5 \mathrm{mg} / \mathrm{l}$ transferrin and $5 \mu \mathrm{g} / \mathrm{l}$ sodium selenite (Sigma-Aldrich, St Louis, MO). Depending on treatment, drugs were added to the medium and metanephroi were incubated at $37^{\circ} \mathrm{C}$ and $5 \% \mathrm{CO}_{2}$ for $24 \mathrm{~h}$. For gentamicin, drug concentrations of 3,30 , and $300 \mu \mathrm{mol} / \mathrm{l}$ were chosen. Ceftazidime and meropenem were both tested at 20,200, and 2,000 $\mu \mathrm{mol} / \mathrm{l}$. Concentration levels were calculated as follows: The clinical pediatric dosing was used and corrected for plasma binding and volume of distribution as detailed in Table 1 (26-32). In addition, concentrations at a factor of 10 above and below were studied to take calculation uncertainties into account and to investigate a potential dose response relationship.

All metanephroi from one litter were randomly assigned to one of the treatment groups and a separate no-treatment group that consisted of metanephroi from the same litter was included for each drug. Per litter, one drug was investigated to rule out differences on basis of variability between litters. Multiple litters were studied per drug. 
Table 2. Primer probe sets ${ }^{\mathrm{a}}$ used in this study

\begin{tabular}{ll}
\hline Gene symbol & Primer-probe set \\
\hline Actb & Endogenous control \\
Hmbs & Mm01143545_m1 \\
Fgf8 & Mm00438921_m1 \\
Gdnf & Mm00599849_m1 \\
Sox9 & Mm00448840_m1 \\
Wt1 & Mm00460570_m1 \\
Bmp7 & Mm00432102_m1 \\
Casp3 & Mm01195085_m1 \\
Casp9 & Mm00516563_m1 \\
\hline
\end{tabular}

aFrom Applied Biosystems (Pleasanton, CA).

\section{Metanephric Growth}

Growth of the explanted metanephroi was determined by measuring surface areas expansion in $24 \mathrm{~h}$. Photographs of the metanephroi were obtained with a Canon EOS 1000D camera (Canon, 's Hertogenbosch, The Netherlands) attached to a Zeiss Axiovert 25 microscope (Carl Zeiss, Sliedrecht, The Netherlands) at a total magnification of $12.5 \times$. Background light was set on maximal intensity and a shutter speed of $40 \mathrm{~ms}$ was used. The photographs were taken with a resolution of 10.1 megapixels and surface area size was analyzed with FIJI/ImageJA version $1.45 \mathrm{i}(33)$.

\section{Ureteric Tip Imaging}

Whole mount immunostaining of the metanephroi was performed to visualize the ureteric tree after $24 \mathrm{~h}$ of culture. The metanephroi were fixed in ice-cold methanol for $10 \mathrm{~min}$ and washed with phosphatebuffered saline (PBS) for $15 \mathrm{~min}$. Subsequently, the metanephroi were incubated in PBS containing 2\% bovine serum albumin for $12 \mathrm{~h}$ to block non-specific binding. After washing with PBS containing $1 \%$ Triton X-100 (PBS-T), metanephroi were incubated with an antibody against calbindin-D28k (Sigma Aldrich), diluted 1:100 in PBS-T, for $24 \mathrm{~h}$. Again after washing with PBS-T, incubation with an Alexa 488 IgG antibody (Invitrogen, Eugene, OR) was performed at a dilution of 1:300 in PBS containing 2\% bovine serum albumin for $24 \mathrm{~h}$. All incubation and washing steps were performed at $4{ }^{\circ} \mathrm{C}$. After a final washing step in PBS-T of $15 \mathrm{~min}$, the metanephroi were mounted on a slide in mounting medium (Dako, Carpinteria, CA) and sealed using paraffin.

For each metanephros, ureteric branching was visualized by confocal laser scanning microscopy using a Leica TCS SP2 microscope. Optical sectioning was performed at a $4 \mu \mathrm{m}$ interval at a magnification of $10 \times$. Per metanephros, between 18 and 30 images were acquired with a resolution of $1,024 \times 1,024$ pixels. Subsequently, the amount of ureteric tips was counted with the multipoint tool in FIJI. A tip was defined as an end point of the whole branching structure that did not show any signs of branching.

\section{Gene Expression Analysis}

RNA was isolated from the metanephroi by combining the Trizol extraction method with the NucleoSpin RNA II isolation kit (Machery-Nagel, Düren, Germany). The methanephroi were suspended in Trizol (Invitrogen, Carlsbad, CA) and incubated for approximately $30 \mathrm{~min}$ with occasional vortexing. After addition of chloroform (Merck), the samples were incubated on ice for $5 \mathrm{~min}$ and centrifuged at $14,000 \mathrm{~g}, 4^{\circ} \mathrm{C}$ for $15 \mathrm{~min}$. The aqueous phase was added 1:1 to $70 \%$ ethanol to adjust binding conditions and loaded on the Nucleospin column. Further purification was performed according to the manufacturers protocol. RNA concentration and quality was assessed with the Nanodrop 2000c spectrophotometer (Thermo Fisher Scientific, Waltham, MA).

Complementary DNA was generated on a Biozym MJ Research PTC-200 Peltier thermal cycler using random primers (Promega, Madison, WI), oligo dT (Promega) and M-MLV reverse transcriptase (Invitrogen). mRNA levels of Wt1, Sox9, Bmp7, Fgf8, Gdnf, Casp3, and Casp 9 were measured by quantitative PCR (qPCR) with Actb and Hmbs as internal standards.

qPCR was performed on a Biorad CFX96 using the gene expression mix and hydrolysis probes (Table 2) as ordered from Applied Biosystems (Pleasanton, CA). Delta-delta CT values were investigated and an up or downregulation of factor 2 was considered biologically relevant.

\section{Statistics}

Metanephric surface expansion at $24 \mathrm{~h}$ and metanephric surface area at explantation were both investigated for correlation with ureteric bud tip development and tested with a Pearson's correlation coefficient.

Comparison of ureteric bud tip development for each treatment was investigated by one-way analysis of variance followed with Dunnett as post hoc. For both analysis, statistical significance was investigated at the $\alpha=0.05$ level.

\section{ACKNOWLEDGMENTS}

We thank the Department of Pathology, Radboud University Medical Center, for providing the microscopy facilities and especially Irene Otte-Holler for her help with confocal imaging.

\section{STATEMENT OF FINANCIAL SUPPORT}

This project was supported by a Kolff grant to MFS (KJPB.08.06) from the Dutch Kidney Foundation, Bussum, The Netherlands.

Disclosure: There is no conflict of interest to disclose.

\section{REFERENCES}

1. Schreuder M, Delemarre-van de Waal H, van Wijk A. Consequences of intrauterine growth restriction for the kidney. Kidney Blood Press Res 2006;29:108-25.

2. The Netherlands Perinatal Registry. Registry Perinatal care in the Netherlands 2005. Utrecht, the Netherlands: The Netherlands Perinatal Registry, 2008.

3. Gilbert T, Lelievre-Pegorier M, Malienou R, Meulemans A, MerletBenichou C. Effects of prenatal and postnatal exposure to gentamicin on renal differentiation in the rat. Toxicology 1987;43:301-13.

4. Gilbert T, Lelievre-Pegorier M, Merlet-Benichou C. Long-term effects of mild oligonephronia induced in utero by gentamicin in the rat. Pediatr Res 1991;30:450-6.

5. Gilbert T, Nabarra B, Merlet-Bénichou C. Light- and electron-microscopic analysis of the kidney in newborn rats exposed to gentamicin in utero. Am J Pathol 1988;130:33-43.

6. Schwedler SB, Gilbert T, Moreau E, Striker LJ, Merlet-Bénichou C, Striker GE. Nephrotoxin exposure in utero reduces glomerular number in sclerosis-prone but not sclerosis-resistant mice. Kidney Int 1999;56:168390.

7. Gilbert T, Cibert C, Moreau E, Géraud G, Merlet-Bénichou C. Early defect in branching morphogenesis of the ureteric bud in induced nephron deficit. Kidney Int 1996;50:783-95.

8. Tune BM. Nephrotoxicity of beta-lactam antibiotics: mechanisms and strategies for prevention. Pediatr Nephrol 1997;11:768-72.

9. Topham JC, Murgatroyd LB, Jones DV, Goonetilleke UR, Wright J. Safety evaluation of meropenem in animals: studies on the kidney. J Antimicrob Chemother 1989;24:Suppl A:287-306.

10. Bax RP, Bastain W, Featherstone A, Wilkinson DM, Hutchison M, Haworth SJ. The pharmacokinetics of meropenem in volunteers. J Antimicrob Chemother 1989;24:Suppl A:311-20.

11. Norrby SR, Newell PA, Faulkner KL, Lesky W. Safety profile of meropenem: international clinical experience based on the first 3125 patients treated with meropenem. J Antimicrob Chemother 1995;36:Suppl A:207-23.

12. Chan SK, Riley PR, Price KL, et al. Corticosteroid-induced kidney dysmorphogenesis is associated with deregulated expression of known cystogenic molecules, as well as Indian hedgehog. Am J Physiol Renal Physiol 2010;298:F346-56.

13. Sebinger DD, Unbekandt M, Ganeva VV, Ofenbauer A, Werner C, Davies JA. A novel, low-volume method for organ culture of embryonic 


\section{Articles $\mid$ Bueters et al.}

kidneys that allows development of cortico-medullary anatomical organization. PLoS ONE 2010;5:e10550.

14. Costantini F. GDNF/Ret signaling and renal branching morphogenesis: From mesenchymal signals to epithelial cell behaviors. Organogenesis 2010;6:252-62.

15. Rosines $\mathrm{E}$, Johkura $\mathrm{K}$, Zhang $\mathrm{X}$, et al. Constructing kidney-like tissues from cells based on programs for organ development: toward a method of in vitro tissue engineering of the kidney. Tissue Eng Part A 2010;16:244155.

16. Srinivas S, Goldberg MR, Watanabe T, D’Agati V, al-Awqati Q, Costantini F. Expression of green fluorescent protein in the ureteric bud of transgenic mice: a new tool for the analysis of ureteric bud morphogenesis. Dev Genet 1999;24:241-51.

17. Kent AL, Douglas-Denton R, Shadbolt B, et al. Indomethacin, ibuprofen and gentamicin administered during late stages of glomerulogenesis do not reduce glomerular number at 14 days of age in the neonatal rat. Pediatr Nephrol 2009;24:1143-9.

18. Lelievre-Pegorier M, Gilbert T, Sakly R, Meulemans A, Merlet-Benichou C. Effect of fetal exposure to gentamicin on kidneys of young guinea pigs. Antimicrob Agents Chemother 1987;31:88-92.

19. Richardson L, Venkataraman S, Stevenson P, et al. EMAGE mouse embryo spatial gene expression database: 2010 update. Nucleic Acids Res 2010;38(Database issue):D703-9.

20. Ortiz LA, Quan A, Weinberg A, Baum M. Effect of prenatal dexamethasone on rat renal development. Kidney Int 2001;59:1663-9.

21. Perantoni AO, Timofeeva O, Naillat F, et al. Inactivation of FGF8 in early mesoderm reveals an essential role in kidney development. Development 2005;132:3859-71.

22. Grieshammer U, Cebrián C, Ilagan R, Meyers E, Herzlinger D, Martin GR. FGF8 is required for cell survival at distinct stages of nephrogenesis and for regulation of gene expression in nascent nephrons. Development 2005;132:3847-57.

23. Dudley AT, Godin RE, Robertson EJ. Interaction between FGF and BMP signaling pathways regulates development of metanephric mesenchyme. Genes Dev 1999;13:1601-13.

24. Araki T, Hayashi M, Nakanishi K, Morishima N, Saruta T. Caspase-9 takes part in programmed cell death in developing mouse kidney. Nephron Exp Nephrol 2003;93:e117-24.

25. Nathanson S, Moreau E, Merlet-Benichou C, Gilbert T. In utero and in vitro exposure to beta-lactams impair kidney development in the rat. J Am Soc Nephrol 2000;11:874-84.

26. Dutch Health Institute. Meropenem, 2013. (http://www.fk.cvz.nl/ preparaatteksten $/ \mathrm{m} /$ meropenem.asp.)

27. Lam YW, Duroux MH, Gambertoglio JG, Barriere SL, Guglielmo BJ. Effect of protein binding on serum bactericidal activities of ceftazidime and cefoperazone in healthy volunteers. Antimicrob Agents Chemother 1988;32:298-302.

28. van den Anker JN, Schoemaker RC, Hop WC, et al. Ceftazidime pharmacokinetics in preterm infants: effects of renal function and gestational age. Clin Pharmacol Ther 1995;58:650-9.

29. Craig WA. The pharmacology of meropenem, a new carbapenem antibiotic. Clin Infect Dis 1997;24:Suppl 2:S266-75.

30. Meropenem: pediatric drug information. In: Basow D, ed. UpToDate Waltham, MA, 2013.

31. Dutch Health Institute. Gentamicin, 2013. (http://www.fk.cvz.nl/ preparaatteksten/g/gentamicine $\% 20$ (systemisch).asp.)

32. Dutch Health Institute. Ceftazidime, 2013. (http://www.fk.cvz.nl/ preparaatteksten/c/ceftazidim.asp.)

33. Schneider CA, Rasband WS, Eliceiri KW. NIH Image to ImageJ: 25 years of image analysis. Nat Methods 2012;9:671-5. 